\title{
Alternative Antimicrobial Formula for Plant Protection
}

\author{
Steliana RODINO, Marian BUTU*, Alina BUTU \\ National Institute of Research and Development for Biological Sciences, Bucharest, Romania, Splaiul Independentei 296, P.O. Box \\ 17-16, 060031, Bucharest, Romania \\ *corresponding author, e-mail: marian_butu@yahoo.com
}

Bulletin USAMV series Agriculture 75(1)/2018

Print ISSN 1843-5246; Electronic ISSN 1843-5386

DOI 10.15835/buasvmcn-agr: 002017

\begin{abstract}
The European Strategy regarding plant protection products encourages the research for solutions which strongly contribute to the agro-ecological transition. They should be able to provide effective and efficient products for both farmers and consumers, reducing the pesticides use. Previous studies reported that extracts prepared from different plant species are capable of inhibiting in vitro development of fungal phytopathogens. The aim of this research was the study of the antimicrobial activity of a plant protection formula composed by a mix of plant extracts. The final scope was establishing a proper treatment timing strategy for controlling primary infections. Therefore, the effectiveness of the mixed formula containing Humulus lupulus and Urtica dioica against Alternaria sp., the cause of early blight attacking several vegetable species, was studied. Experimentation plan was performed using standard methods for antimicrobial testing. The potential and limitations of plant extracts used to control early blight were discussed. The results obtained have potential practical applicability and are directed to the development of environmentally viable solutions for the design of bioproducts for plant protection. Better knowledge on this topic will help optimizing crop protection strategy, ensuring sustainable agricultural development.
\end{abstract}

Keywords: phyto-protection formulas, antimicrobial activity, Humulus lupulus, Urtica dioica

\section{Introduction}

Plant fungal pathogens are the cause of important loss in horticultural production worldwide. There are numerous studies reporting the occurrence and methods of control and treatment for diseases like late blight of potatoes of tomatoes, ergot of rye and wheat, damping off seedlings for vegetables, rusts, downy and powdery mildews, brown spot etc., all causing severe damages to agricultural production. It is considered that most of the plant diseases (more than $80 \%$ ) occurring in agricultural and horticultural systems are triggered by plant fungal pathogens or fungal-like organisms (Agrios, 2009).

The introduction of synthetic fungicides in agricultural practices reduced the negative impact of many plant fungal diseases and increased the overall yield (Shuping et al., 2017), so it can be concluded that they have effectively done their work in controlling fungal disease, over the decades. Unfortunately, using them repeatedly has resulted in development of fungal resistance and disrupted natural biological systems triggering undesirable effects on non-target organisms (Yoon et al., 2013). Since the 1970s, farmers observed the emergence of pathogens resistance against fungicides (Shuping et al., 2017). Therefore, aiming to find novel crop protection solutions, studies regarding the possible exploitation of biomaterials such as natural pesticides were performed (Yoon et al., 2013).

The EU set rules for sustainable use of pesticides towards reducing the risk and impact of pesticide use on people's health and the environment through Directive 2009/128/EC. The present European recommendations regarding the plant protection products encourages research for solutions which can contribute to the agro- 
ecological transition, thus providing effective and efficient products for both farmers and consumers and reducing the use of synthetic fungicides. Plant protection products should contain at least one approved active substance, including microorganisms, pheromones and botanical extracts.

Plant extracts and microbial cultures serve as sources of various biologically active compounds, including biofungicides (Arsene et al., 2015), with potential to suppress the development of plant diseases. Testing these extracts for their activity against the pathogenic fungiattacking horticultural crops represents a promising research direction, as broad-spectrum biopesticides can be developed.

Alternaria spp. causes leaf spots and blight on a large variety of agricultural and horticultural crops such as: tomato (Lycopersicon esculentum), potato (Solanum tuberosum), carrot (Daucus carota), cauliflower (Brassica oleracea var. botrytis), broccoli (Brassica oleracea - Botrytis Group), cabbage (Brassica oleracea var. capitata), peppers (Capsicum annuum), beans (Pisum sativum), apple (Malus domestica), peach (Prunus persica) and citrus species. Moreover, Alternaria spp. can also attack a several weeds and ornamental plants (Rodino et al., 2014).

The aim of this paper was the study of the antimicrobial activity of a plant protection formula composed by a mix of plant extracts, for establishing a proper treatment timing strategy for controlling primary infections. The effectiveness of the mixed formula containing Humulus lupulus and Urtica dioica against Alternaria sp., the cause of early blight attacking several vegetable species, was studied.

\section{Materials and methods}

\section{Preparation of antimicrobial solution}

The antimicrobial solution tested represented a mix formula of the following plant extracts: Humulus lupulus female inflorescences and Urtica follium. The plant material was collected from agricultural fields in Southern part of Romania. The single plant extracts were obtained by ultrasonication (for 30 minutes, power 34 watts, $35^{\circ} \mathrm{C}$ ) followed by $24 \mathrm{~h}$ maceration. The solvent used for extraction was ethanol $70 \%$. The ratio between dried plant material (powder) and solvent was 1:5. The extracts were filtered through Whatman No 1. filter paper and the combined filtrate was concentrated to dryness by rotary evaporator. For the antimicrobial tests, the desired dilutions were prepared by dissolving with sterile double distilled water.

\section{Inoculum preparation}

Pure cultures of Alternaria sp. were maintained on PDA slants and stored at $4{ }^{\circ} \mathrm{C}$ throughout the study period. Fresh culture was maintained on potato dextrose agar (PDA) amended with $250 \mu \mathrm{g}$ $\mathrm{mL}^{-1}$ chloramphenicol, for 10 to 14 days at $20 \pm 2$ ${ }^{\circ} \mathrm{C} .10 \mathrm{~mL}$ of sterile distilled water was added to each Petri-dish containing a culture of Alternaria $s p$., colonies were scrapped with a sterile loop and spores were collected. The spore suspension obtained was filtered through cheese cloth. The inoculum concentration was adjusted to $10^{6} \mathrm{~mL}^{-1}$.

\section{Screening of antimicrobial solution in vitro efficacy against early blight causal agent}

To assess the mycelial growth inhibition of Alternaria sp. caused by the antimicrobial solution prepared, the radial growth assay (or poisoned food method) was used as described earlier (Rodino et al., 2015).

Bravo, commercial fungicide, was used as the positive control in the in vitro tests. Control treatment was considered the Petri dish without adding fungicide or plant extract. The Petri dishes were incubated for 7 days at $20 \pm 2{ }^{\circ} \mathrm{C}$. The mycelial growth was measured as mean of two perpendicular diameters. The efficacy of the treatment was determined as percentage of the growth inhibition of the mycelium relative to the control treatment, (I) and was calculated according to the following formula:

I (\%) = (1-Dt / Dc)*100 (\%), where, $\mathrm{Dc}=$ average diameter of the negative control, $\mathrm{Dt}=$ average diameter of the treated cultures or the positive control.

\section{Detached leaves assay}

The tomato leaves used in experiments were taken from seedlings grown in greenhouse under controlled conditions, in sterilized soil. For the collection of leaves, healthy plants, untreated with synthetic fungicides, from 8 to 9 weeks old were used. Counting from the top of the plant, it was detached from the stem, third to fifth composite leaf and was used in tests within 2 hours from the harvesting moment. Single leaves were placed 


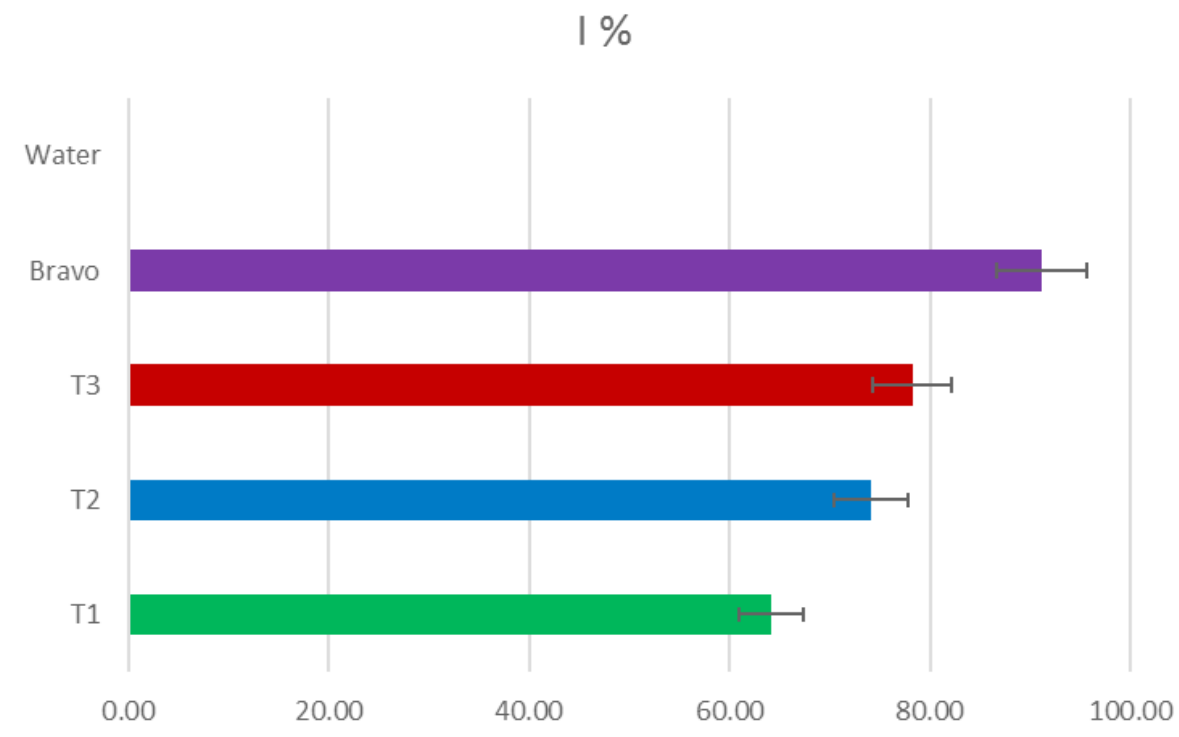

Figure 1. The antifungal activity of antimicrobial formula against Alternaria sp. (results are presented as the mean of triplicate determinations \pm standard deviation)

with the abaxial side upwards on water agar (15 g/L agar) in Petri dishes.

From this moment two different strategies were performed as follows:

Treatment before inoculation

The detached leaves were treated with the antimicrobial formula previously used in the preliminary antimicrobial tests, performed in vitro, in Petri dishes, on growth medium. The working solutions were brought to a final concentration of $15 \%$ (S1) and $10 \%$ (S2).

The treatment was applied to 10 leaflets by spraying the solution onto the upper surface of the leaf. Positive control was Bravo solution at a concentration of $0.2 \%$. This fungicide is commonly used in horticultural practices to prevent infection of Alternaria spp. on tomato, potato and eggplant crops. The negative control was a sterile distilled water solution.

\section{Treatment after inoculation.}

Basically, this strategy follows the same protocol as the first one. The leaves were first inoculated and then treated with antimicrobial formula (S3 and S4) or control solutions. Positive control was Otriva solution, a fungicide applied to stop the development of early blight in horticultural crops.

The sealed Petri dishes were kept in a controlled environment for 7 days $\left(20^{\circ} \mathrm{C}, 16 \mathrm{~h}\right.$ light period). After one week, the disease development was observed and the severity of symptoms caused by infection with Alternaria sp. was evaluated on an adapted scale previously described by Akhtar et al, 2012. The intensity of disease is following an evaluation score chart from $0-5(0=$ no visible signs of disease $-0 \%, 1=$ few lesion spots $-10 \%$, $2=$ about $25 \%$ of the total leaf area is infected, $3=$ about $50 \%$ of the total leaf area is blighted, 4 = about $75 \%$ of the total leaf area is affected, 5 $=$ the leaves are necrotic, dehydrated, covered by injuries on more than $75 \%$ of their surface).

\section{Results and discussions}

According to the bibliographical study and to previous investigations of the authors on the use of plant extracts with antimicrobial activity on plant pathogens, in the first part of the experiments were used three different concentrations of the plant extract in the growth medium. Although the response of the fungal pathogen to each concentration was different over the control, generally growth inhibition increased with the concentration, on the tested range. Thus, on a concentration of $10 \%$ (T3) in the growth medium, was found that the antimicrobial formula presented fungistatic activity. Lowering the concentration to $5 \%$ (T2) it could be observed that although the mycelium growth debuted in the same day with the untreated control, it had a considerable backlog. On the seventh day after inoculation, on the treated plate (5\%) the mycelium diameter reached $20 \mathrm{~mm}$ while the untreated control 


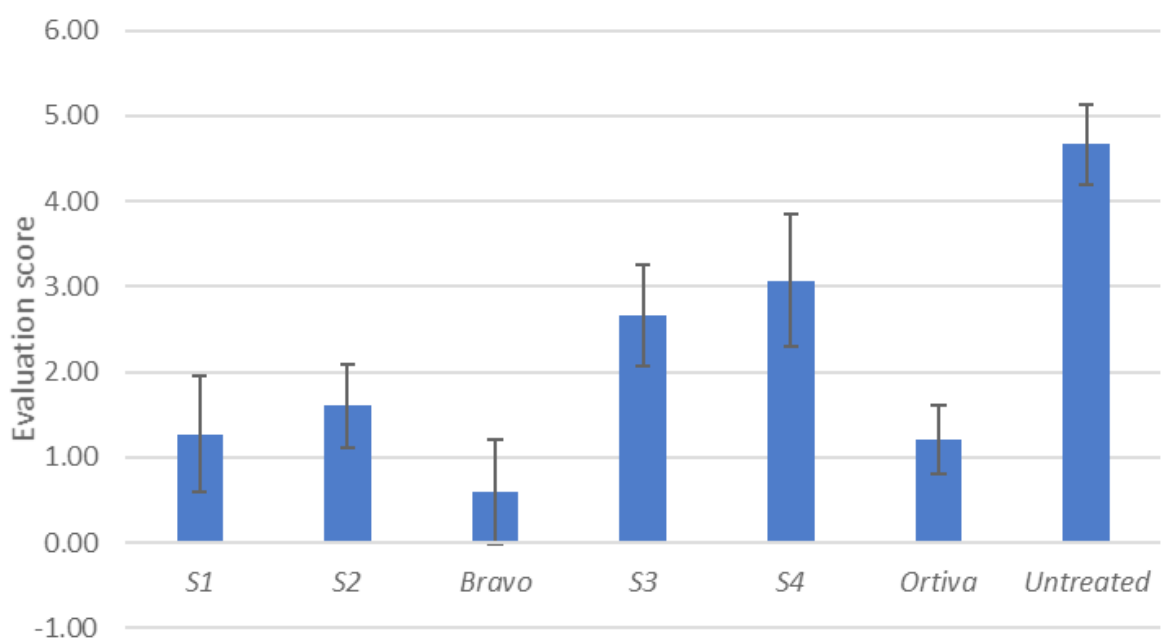

Figure 2. Evaluation score observed after detached leaves assay for antimicrobial formula and controls

reached the margins of the Petri plate measuring $70 \mathrm{~mm}$. This slower trend was maintained in the case of a concentration of $2 \%$ (T1).

Taking into account these results, it was decided to continue with evaluation of the antimicrobial formula effect against Alternaria sp., with the concentration starting from minimum 10 $\%$ (T3), which inhibited the mycelium growth by $74.16 \%$ (Fig. 1).

For the evaluation of the plant response, the infestation of tomato leaves with Alternaria $s p$. was performed following a method previously described in the literature (Cowley et al., 2012; Foolad et al., 2014; Lohith et al., 2011) with some changes.

The detached leaves test is a generally accepted experimental model, being a technique commonly used to assess the resistance of plant pathogens to different products or plant varieties under laboratory conditions. The detached leaves test proved to be a practical solution because of its simplicity, which can substantially reduce both the costs and duration of a screening program as previously reported by other authors (Irzhansky et al., 2006; Nelson, 2006).

S1 (15 \% concentration) and S2 (10\% concentration) and Bravo (Syngenta) were tested before inoculation, and S3 (15\% concentration), S4 (10\% concentration) and Ortiva (Syngenta) after inoculation.

As can be seen in Fig. 2, treatment as a preventive measure prior to inoculation resulted in a better management of infection caused by Alternaria spp. Although the results obtained with the antimicrobial formula after inoculation were slightly better than untreated control, it can not be said that this formula represents a viable alternative to the fungicide tested. In future experiments, an optimization of the formula will be considered, when applying the solution as a treatment for the already installed disease.

Early blight is an economically important foliar fungal disease of Solanaceae crops, representing, the most common disease of the cultivated tomato in areas with frequent rainfall and high humidity. The necrotrophic nature of this plant pathogen can lead to complete defoliation of tomato plants and subsequent yield reductions. Various Solanaceae species can serve as alternate host for overwintering of the pathogen (Panthee et al. 2010), and it would be useful to find a new, innovative solution for the management and control of this disease.

Hops (Humulus lupulus) is perennial plant whose female inflorescences are well-known as bittering agents in the brewing industry. Hop cones, presenting a high content of polyphenolic compounds and acyl phloroglucides, have been used for various medical purposes (sleep disturbances, anxiety, excitability, acne, dysmenorrhea) over the time. Other authors studied the hops extracts for the determination of its polyphenols, essential oils and resin content and for the DPPH scavenging activity (Arsene et al., 2015). Ethanolic extract from Humulus lupulus inflorescences showed antioxidant activity in a dose-dependent manner. Moreover, the hops extract exhibited antifungal activities against 
food spoilage fungi (Aspergillus niger, A. flavus, Penicillium citrinum, P. chrysogenum and Rhizopus oryza) (Niknejad et al. 2014)

Antimicrobial potential and other beneficial effects Urtica species was also reported by other authors (Hadizadeh, et al. 2009; Gülçin et al., 2004) on a variety of pathogens. A recent study reported the relatively high amount of phenolic and flavonoid contents of the aqueous extract obtained from leaves of Stinging nettle (Fattahi et al., 2014). Körpe et al., (Körpe et al., 2013) observed high antibacterial activity of Urtica spp. seed extracts against both food- and plantborne pathogens. Among the plants tested (nettle, oleander, colocynth and konar) Hadizadeh et al., concluded that the nettle was among the most effective against plant fungal pathogens Alternaria alternata and Rhizoctonia solani.

\section{Conclusions}

The results obtained have potential practical applicability and are directed to the development of environmentally viable solutions for the design of bioproducts for plant protection. The mixed antimicrobial formula was obtained from an original combination of plant material (hops and nettle), with antifungal effects already proven against phytopathogens. Optimizing this formula can lead to the establishment of a plant-friendly bioproduct recipe for farmers who want to implement environmentally friendly solutions in line with European requirements.

Better knowledge on this topic will help optimizing crop protection strategy and ensuring sustainable agricultural development.

Acknowledgements: This work was supported by a grant of the Romanian National Authority for Scientific Research and Innovation, CNCS/ CCCDI - UEFISCDI, project number PN-III-P2-2.1PED-2016-1544, contract number 14PED / 2017, within PNCDI III and Program NUCLEU PN 16 / 2016.

\section{References}

1. Akhtar KP, Saleem MY, Asghar M, Sarwar N, Elahi MT (2012). Resistance of Solanum species to Phytophthora Infestans evaluated in the detached-leaf and whole-plant assays. Pak. J. Bot., 44(3): 1141-1146.

2. Arsene L, Rodino S, Butu A, Petrache P, Iordache O, Butu $M$ (2015). Study on antimicrobial and antioxidant activity and phenolic content of ethanolic extract of Humulus lupulus, Farmacia, 63(6): 851-857.
3. Agrios GN (2009). Plant pathogens and disease: general introduction. Elsevier Inc., University of Florida, Gainesville, FL, USA.

4. Cowley RB, Luckett DJ, Harper JDI, Ash GJ (2012). Development of a reliable and rapid detached leaf assay to detect resistance to the fungal disease phomopsis leaf blight, caused by Diaporthe toxica, in Lupinus albus. Canadian Journal of Plant Pathology, 34(3): 401-409.

5. Desta M, Yesuf M (2015). Efficacy and Economics of Fungicides and their Application Schedule for Early Blight (Alternaria solani) Management and Yield of Tomato at South Tigray, Ethiopia. J Plant Pathol Microb 6:268.

6. Fattahi S, Zabihi E, Abedian Z, Pourbagher R, Motevalizadeh Ardekani A, Mostafazadeh A, Akhavan-Niaki H (2014). Total Phenolic and Flavonoid Contents of Aqueous Extract of Stinging Nettle and In Vitro Antiproliferative Effect on Hela and BT-474 Cell Lines. International Journal of Molecular and Cellular Medicine, 3(2): 102-107.

7. Foolad MR, Sullenberger MT, Ashrafi H (2014). DetachedLeaflet evaluation of tomato germplasm for late blight Resistance and its correspondence to field and greenhouse screenings. Plant Disease 99(5): 718-722.

8. Gülçin I, Küfrevioglu OI, Oktay M, Büyükokuroglu ME (2004). Antioxidant, antimicrobial, antiulcer and analgesic activities of nettle (Urtica dioica L.). J Ethnopharmacol. 90:205-215.

9. Hadizadeh I, Peivastegan B, Kolahi M (2009). Antifungal activity of nettle (Urtica dioica L.), colocynth (Citrullus colocynthis L. Schrad), oleander (Nerium oleander L.) and konar (Ziziphus spina-christi L.) extracts on plants pathogenic fungi. Pakistan J Biolog Sci. 12(1):58-63.

10. Irzhansky I, Cohen Y, (2006). Inheritance of resistance against Phytophthora infestans in Lycopersicon pimpenellifolium L3707. Euphytica. 149(3): 309-316.

11. Körpe DA, İşer OD, Sahin FI, Cabi E, Haberal M (2013). High-antibacterial activity of Urtica spp. seed extracts on food and plant pathogenic bacteria. International Journal of Food Sciences and Nutrition. 64 (3): 355-362.

12. Lohith MR, Chandrashekar Reddy K, Venkata Ramana C, Venkata Rao P, Ravendra Reddy K, Lokanadha Reddy D (2011). Screening of tomato genotypes against early blight (Alternaria solani) by detached leaf method. Acta Hortic. 914: 465-468.

13. Nelson HE (2006). Bioassay to detect small differences in resistance of tomato to late blight according to leaf age, leaf and leaflet position, and plant age. Australasian Plant Pathology 35(3): 297-301.

14. Niknejad F, Mohammadi M, Khomeiri M, Razavi SH, Alami M (2014). Antifungal and Antioxidant Effects of Hops (Humulus lupulus L.) Flower Extracts, Advances in Environmental Biology, 8(24): 395-401.

15. Panthee DR, Chen F (2010). Genomics of Fungal Disease Resistance in Tomato. Current Genomics. 11(1):30-39.

16. Rodino S, Butu M, Petrache P, Butu A, Cornea CP (2014) Antifungal activity of four plants against Alternaria alternata. Scientific Bulletin. Series F. Biotechnologies, XVIII: 60-65. 
17. Shuping DSS, Eloff JN (2017) The use of plants to protect plants and food against fungal pathogens: A review. African Journal of Traditional, Complementary, and Alternative Medicines. 14(4):120-127.

18. Rodino S, Butu M, Fidler G, Butu A, Negoescu G, Cornea CP (2015) Research regarding the antimicrobial activity of plant extracts, Bulletin UASVM Animal Science and Biotechnologies, 72(1):54-58.

19. Yoon MY, Cha B, Kim JC (2013). Recent trends in studies on botanical fungicides in agriculture. The Plant Pathology Journal. 29(1):1-9. 